\title{
Chaoslike States Can Be Expected Before and After Logistic Growth
}

\author{
THEODORE MODIS and ALAIN DEBECKER
}

ABSTRACT

Instabilities associated with population growth can be simulated by putting the logistic growth curve in a discrete form. In contrast to the usual derivation of chaos, which can only explain instabilities at the top of the curve, this method can also account for fluctuations during the early phases of the niche-filling process. Precursors, a steep initial rise, and final instabilities can all be interrelated. Industrial examples are given of logistic growth alternating with periods of chaotic fluctuations.

\section{Historical Background}

The mathematics involving discrete iterative processes is becoming increasingly important. It may well prove more useful to mankind than formulations of analytic functions of continuous variables. This is not only a consequence of the increasingly dominant role played by computers that perform mathematical operations through iterative numerical techniques. Physicists have shown that everything in nature is quantized, from matter to light. Spectacular consequences of putting natural-law descriptions in a discrete form have been the subjects of chaos and fractals. In their book The Beauty of Fractals [1] featuring over 100 eye-pleasing, mind-boggling, color maps, Peitgen and Richter cast the logistic differential equation

$$
\frac{1}{X} \frac{\mathrm{d} X}{\mathrm{~d} t}=\alpha(M-\mathrm{x})
$$

where $X$ is the population, and $\alpha$ and $M$ are constants, into the discrete form

$$
X_{n+1}=r X_{n}\left(1-X_{n}\right)
$$

where $X_{n}$ is the population at the $n$th iteration, and $r$ is a constant.

Equation 1 gives rise to logistic growth that follows elegant-shaped $\mathrm{S}$ curves. Equa-

THEODORE MODIS is a physicist who has carried out experiments in elementary particles for over 15 years at CERN, in Geneva, Switzerland; at Brookhaven National Laboratory, Upton, New York; and at NEVIS Laboratories, Irvington-on-Hudson, New York. He has also been on the teaching faculties of Columbia and Geneva Universities. ALAIN DEBECKER is a mathematician and a management science consultant, teaching Quantitative Methods for management at Lyon University. 
tion 2 leads to states of chaos and the fascinating world of fractals. The bridge between continuous and discrete formulations spans 150 years of developments in mathematics. It starts with Verhulst [2] finishes with Mandelbrot [3] and intricately links order with chaos.

The work reported here also links logistic growth to erratic fluctuations, but through a somewhat different approach. Our motivation was not to study chaos. We were simply trying to find ways to speed up a computer program. Working for a computer company, one is often spoilt because there are usually unlimited computing resources. Nevertheless, human nature is such that having does not quench greed. When we realized that it required more than a few minutes of real-time computing, for the minimization of a $\chi^{2}$ involving the convolution of two logistic functions, we found the situation unacceptable [4]. Instead of searching for a programming trick that would accelerate the process, we used a mathematical trick. In constructing the $S$ curve, we replaced the analytic function by an iterative process. We obtained the familiar logistic-growth pattern, but, in addition, we observed chaoslike states before and after the steep rise.

This finding came as an ex machina deus because important fluctuations at the extremities of $S$ curves had often been observed and had served as ammunition to critics of logistic-growth applications.

\section{Making an S Curve Discrete}

Maede [5] has shown that logistic growth can be obtained through a simple hyperbola in the recursive relation:

$$
X_{n+1}=\frac{X_{n}}{a X_{n}+b}
$$

where $a$ and $b$ are constants and there is a third constant involved, the starting value $X_{0}$. Such iterative operations produce $S$ curves like the solutions of equation 1 . This approach is different from the one of chaos scientists who first put the differential equation in a discrete form-equation 2 -and then iterate it. We essentially put the solution of the differential equation in a discrete form.

A mathematically equivalent form of equation 3 is the following:

$$
\frac{X_{n}}{M-X_{n}}=k \frac{X_{n-1}}{M-X_{n-1}}
$$

where $M$ is the niche size just as in equation 1 , and $k$ can be linked to the constant $\alpha$ of equation 1 through such a relationship as $k=e^{\alpha M}$. Equation 4 lcads to

$$
\frac{X_{n}}{M-X_{n}}=u k^{n} \quad \text { where } \quad u=\frac{X_{0}}{M-X_{0}}
$$

We see that, with time (that is, as $n$ increases), this quantity converges toward 0 or $+\infty$, depending on $|k|$ IF $|k|>1$, the population $X_{n}$ grows toward $M$, which behaves as an attractor. For $|k|<1$, the population decreases toward 0 , which now becomes the attractor, while $M$ behaves like a repeller. The relationship between successive terms $X_{n}$ can be seen pictorially in Figure 1a. The population curve traces out the familiar $\mathrm{S}$ shape of logistic growth. 

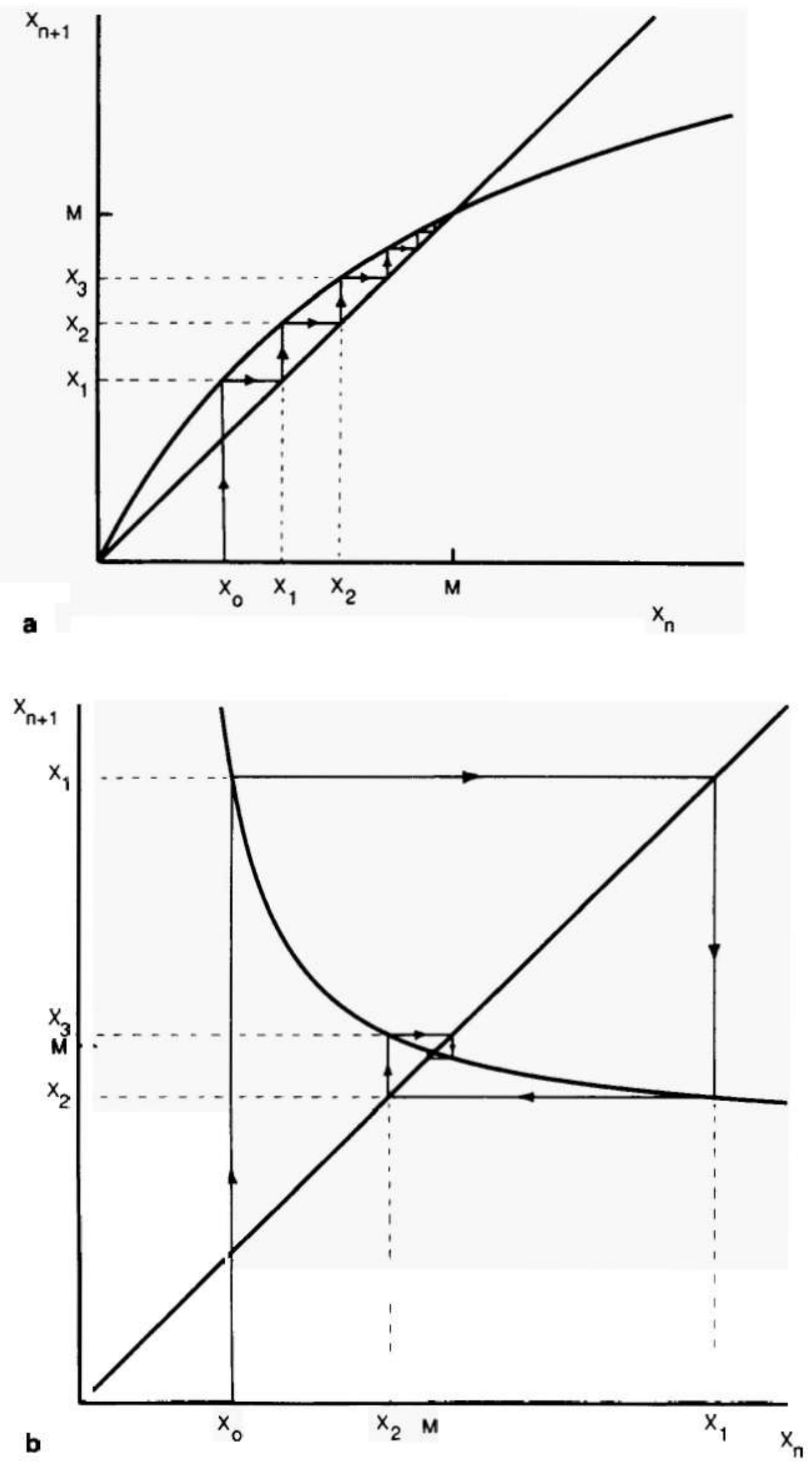

Fig. 1. Iterations of a hyperbola. Graphs of equation 4 (the thin line indicates the sequence of terms): (a) $k>1$, a monotonic approach to the value $M$; and (b) $k<0$, convergence following excursions above and below $M$. 
For equation 4 , it is natural that $k>0$. However, in equation 3, proposed by Maede and used in our iteration, the sign of $\mathrm{k}-\mathrm{a}$ nontrivial formulation in terms of $a$ and $b$ was not something we thought constraining. So we observed the consequences of having $k<0$, namely, oscillations. Successive terms of the sequence $X_{n}$ oscillate around the end value $M$, as indicated in Figure $1 \mathrm{~b}$. The population goes through an overshoot, falls back, and proceeds through oscillations of diminishing amplitude after which it stabilizes at the value $M$.

Negative values for the constant $k$ imply complex values for the constant $\alpha$ of the logistic equation 1. The modulus of $k$ is related to the steepness of the growth while its argument determines the frequency of the oscillations. Complex values for $\alpha$ and $k$ produced the states shown in Figure 2. At the top, we see the beginning of an oscillation. For some values of the parameters, we obtain the rapid oscillations shown in Figure $2 \mathrm{~b}$. At the bottom, we see a case with an irrational period yielding irregular fluctuations. In all cases, the oscillations precede as well as follow the logistic growth.

The states of Figure 2 resemble publicized pictures obtained by chaos scientists via equation 2 [6]. Our approach, however, does not lead to true chaos. The oscillations we observe die out sooner or later; the damping constant can be large, but is not infinite. We have used the hyperbolic function, equation 3, instead of the parabolic one, equation 2. Consequently, our series is a reversible one; from late values, one can reconstruct early ones, which is not the case with true chaos. We can also interpolate the discrete points indefinitely thus recovering a smooth curve, which again is not possible with chaos. The smooth curve that can be recovered from formula 5 is the solution of equation 1, namely, the familiar logistic growth:

$$
X_{n}=\frac{M}{1 \div \mathrm{u}^{-1} \mathrm{k}^{-n}}=\frac{M}{1 \div e^{-\alpha\left(n-t_{0}\right)}}
$$

but with $\alpha$ complex, $t_{0}$ is a simple constant.

Upon approaching the ceiling, the discrete form-Figure $2 \mathrm{c}$-shows erratic instabilities not unlike those observed in chaotic states, but with an additional new feature. Instabilities are now also encountered before the main thrust of the growth.

For true chaos obtained via equation 2 , the amount of irregularity is linked to the magnitude of $r$. As $r$ increases, one witnesses the onset of oscillations first simple, then complicated, and eventually chaos. In our approach, it is the $\arg (k)$ that gives rise to the oscillations, while $|k|$ only relates to the steepness of the growth and the damping constant of the oscillations. The dependence on $k$ is as follows:

- For $\arg (k)=0$, that is, $\alpha$ real, we obtain the classic logistic growth.

- For $\arg (k)=\pi$, that is, $k<0$, we observe regular oscillations alternating above and below $M$.

- For $\arg (k)=(p / q) \cdot 2 \pi$, where $p$ and $q$ are prime integers, the series oscillates with period $q$.

- Finally, when $\arg (k)$ is an irrational multiple of $\pi$, then there is nonrepeating fluctuations resembling true chaos patterns.

Rather than upon crossing a threshold value (as for the parameter $r$ ), our chaos appears for a dense subset of the parameter $k$ impacting the oscillation period. 


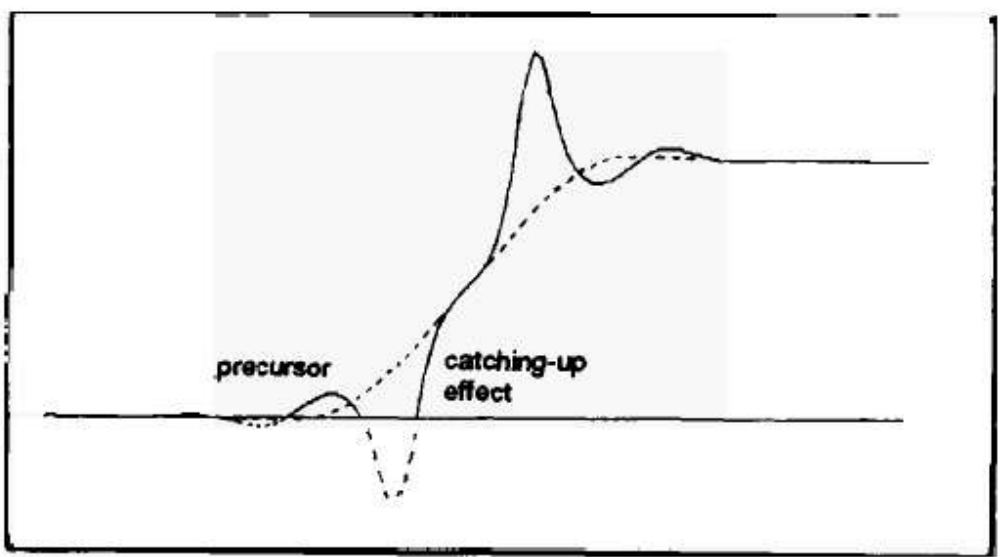

a
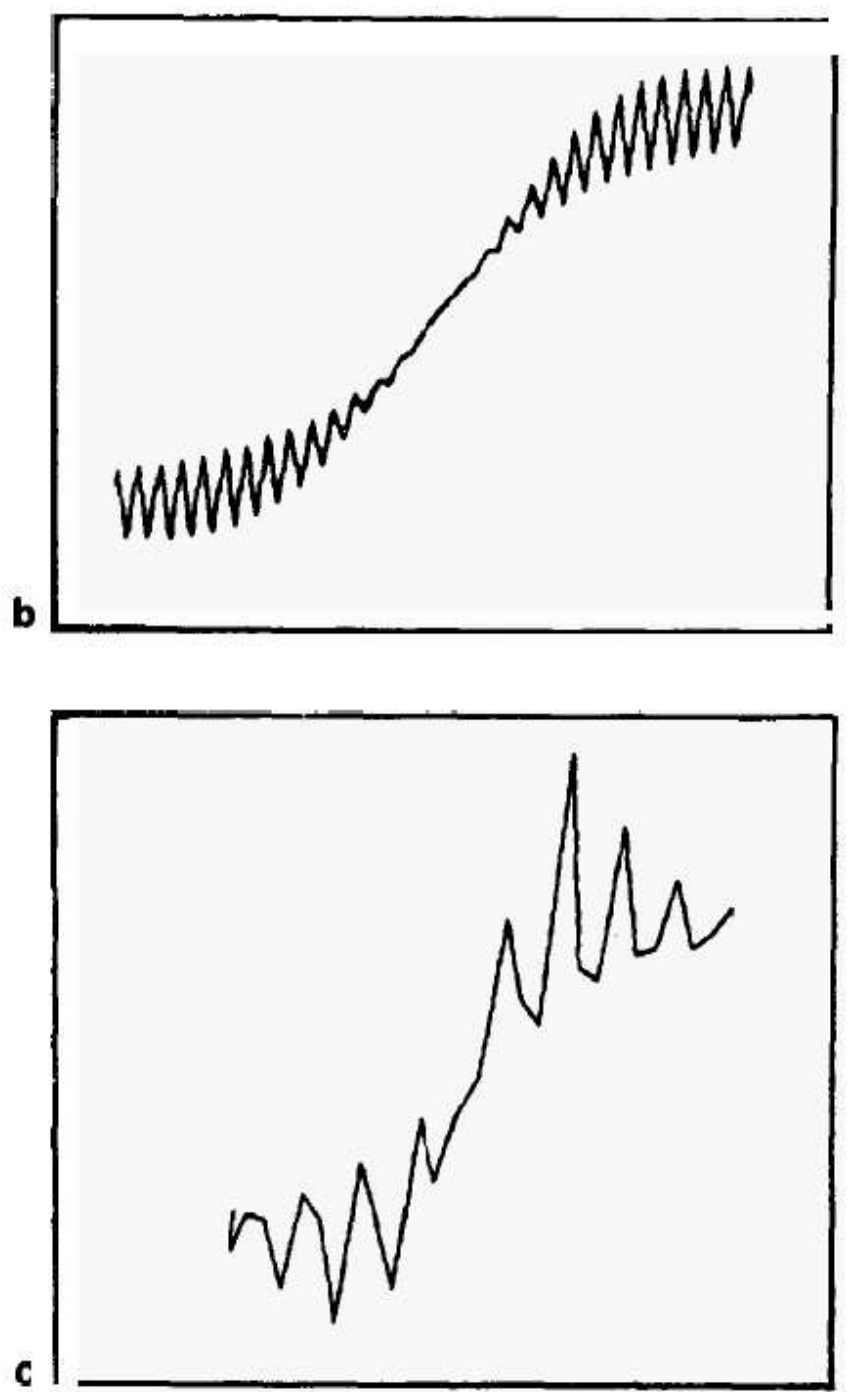

Fig. 2. Making an $\mathbf{S}$ curve discrete. These are examples of the patterns obtained by varying the parameters in the discrete formulation of the logistic growth function. (a) The so-called catching-up effect and the possibility of precursor and overshoot. The line appears smooth because of rapid sampling. (b) Regular oscillations. (c) States resembling chaos. In all cases, similar behavior is observed before as well as after the main growth phase. 
Annual registrations

in millions

a

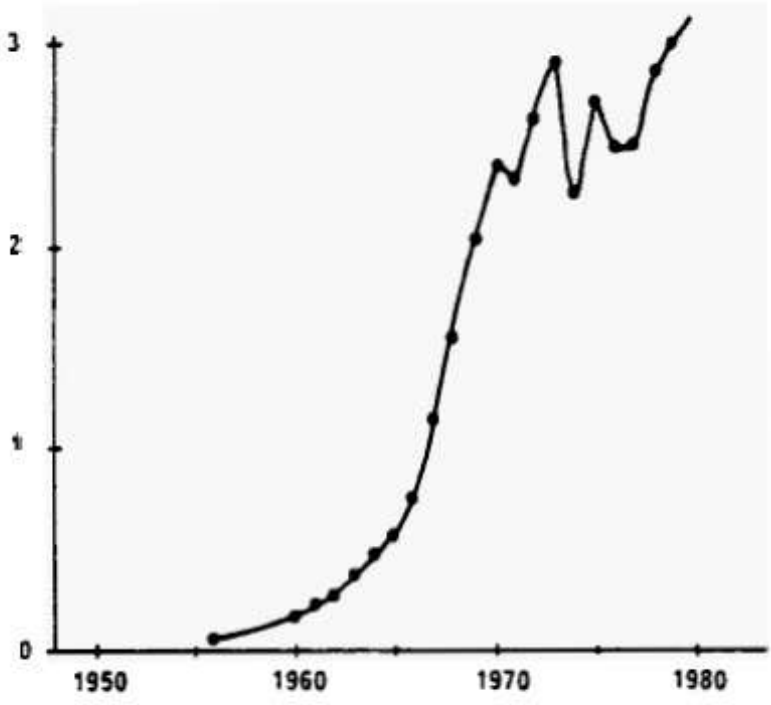

Fig. 3. (a) This graph shows data for new car registrations in Japan as reported by Marchetti [8]. (b) This graph is taken from the article of Montrey and Utterback [9] and shows the annual plywood sales in the United States. In both cases, significant deviations from a logistic pattern appear when the ceiling is approached.

\section{Deviations at Both Extremities of the S Curve}

When chaos studies first diffused through the media, they were welcomed by logisticgrowth advocates who were in need of an explanation for the anomalies they had encountered. The annual rate of a population growth into a new niche had often been seen to follow a logistic pattern that brakes into fluctuations of random character and sizable amplitude just before reaching the ceiling. ' Two such examples are shown in Figure 3, the number of new car registrations in Japan as reported by Marchetti, and the annual sales of plywood in the United States as reported by Montrey and Utterback [9]. Both processes follow a logistic-growth pattern developing significant random fluctuations as the ceiling is approached. Chaos provides a mechanism for explaining these fluctuations.

'Such oscillations have been reported by Elliott Montroll and Wade Badger [7], and later by Cesare Marchetti [8]. 
Billions of tons

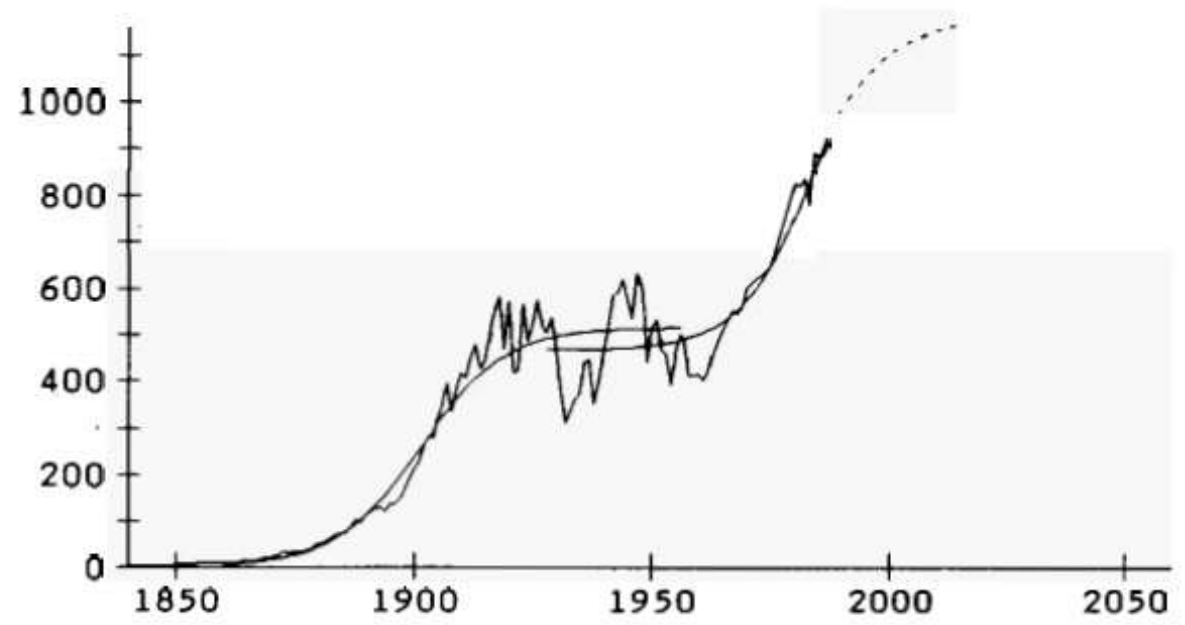

Fig. 4. Annual production of bituminous coal in the United States [10, 11]. The $S$ curves shown are logistic fits to the data for the respective historical periods. The Interim period shows large fluctuations of chaotic nature.

However, there have also been systematic deviations from logistic patterns during the early phases of the growth. These deviations can not be explained by the classic chaos formulation.

Marchetti in his numerous applications of logistic growth has characterized early deviations from $\mathrm{S}$ curves in behavioral terms using such metaphors as "infant mortality" and "catching-up effect." With the former, he refers to unsuccessful competitive growth before it reaches $10 \%$ of the ceiling. This phase can be considered as a trial period, during which survival is at stake and major readjustments may be undertaken. Many young organisms, not properly cared for, die during this period. By analogy, industrial products are often repositioned pricewise and/or performancewise shortly after launching.

Marchetti's second characterization refers to the sudden release of pent-up energy following a "technical" delay in the early evolution of a population. It is manifested as growth at an accelerated rate until the logistic trajectory is reached. Again, there is an industrial analogue, a surge in sales with some product introductions.

The fluctuations preceding the logistic rise in Figure 2 provide an explanation for the early irregularities mentioned above. In Figure 2a, we see the beginning of an oscillation before the $S$ curve develops. Negative excursions of a population have no physical meaning, so what is observed from the perturbation is a precursor and a steep rise toward the logistic path. Marchetti would have said infant mortality followed by a catching-up effect.

Variations in the annual rate of a population can be large, but they do not always imply that we are simply witnessing the chaotic behavior that follows the completion of a niche. The random fluctuations could equally well belong to a new growth process that will fill up a new "market" niche. Such is the case depicted in Figure 4, of bituminous coal production in the United States. ${ }^{2}$ The large oscillations in the annual production of coal between the years 1920 and 1960 may be seen as belonging to the ceiling of the first $\mathrm{S}$ curve as well as to the beginning of the second one. In fact, had we had no

\footnotetext{
${ }^{2}$ Annual data for coal production come from the US Bureau of the Census $[10,11]$.
} 


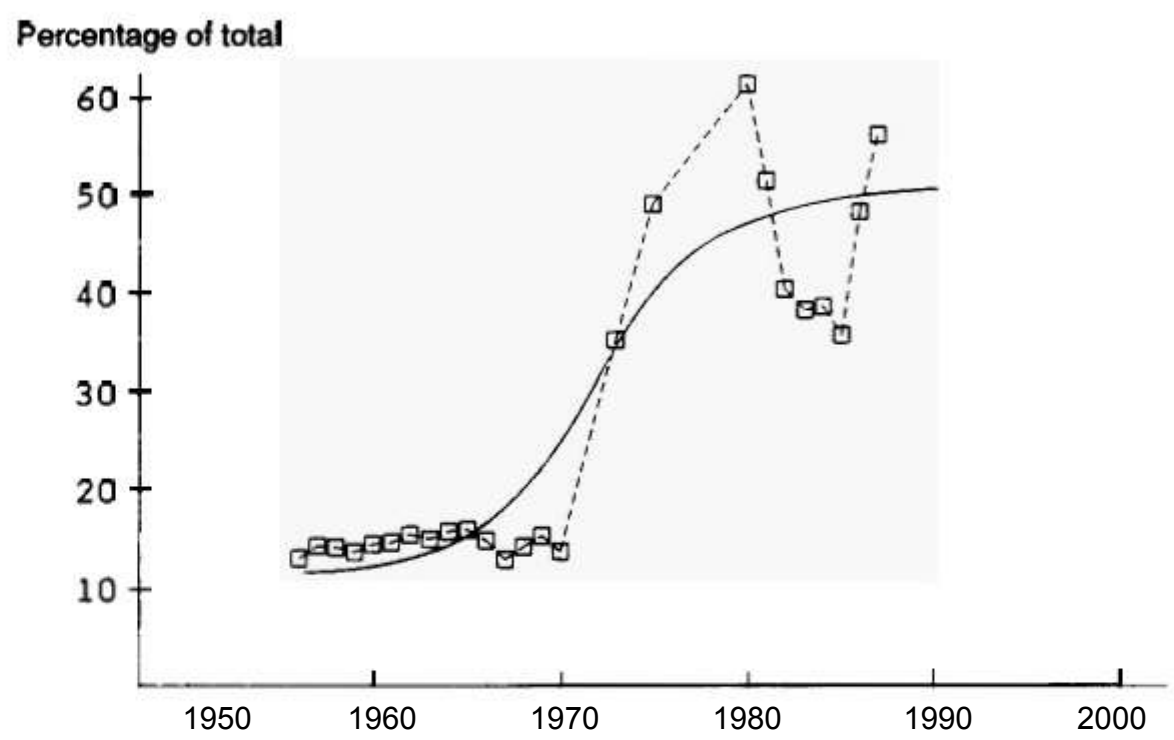

Fig. 5. US oil imports as a percentage of total (imports plus domenstic production). The S-curve shown is an idealized natural-growth process. The large-amplitude oscillations may have been triggered by pent-up demand caused by the pre-1970 restrictions.

historical data before 1950 , we would have observed a deviation between data and curve around 1960 that we would have rightfully interpreted as a catching-up effect.

As can be surmised from Figure $2 \mathrm{a}$, the presence of a precursor should correlate with a steeper initial rise, which in turn leads into an overshoot upon approaching the ceiling. Such a picture is partially depicted by the data on oil imports in the United States shown in Figure $5 .{ }^{3}$ Before 1969 , there was a law limiting the amount of oil imported in the United States to a fixed fraction of the total oil consumption. This shows up in Figure 5 as a flat percentage until 1969. The fact, however, that the quota was consistently met indicates a certain pressure on the constraining law. It was perhaps in response to this pressure that the restriction was lifted in 1970. What followed is a sharp rise in the percentage of imported oil reaching more than $60 \%$ ten years later, falling back to almost $30 \%$ in 1984 and rising significantly afterward. The logistic-growth curve drawn in Figure 5 is an artistic interpretation of what a "natural" niche-filling process would have looked like. The large-size oscillations around the $50 \%$ level might have been triggered by the sharp rise of the early 1970 s, itself a reaction to the long-term quota imposition.

The generalized picture is one of alternating states of logistic growth and chaos. A clear example is the per capital energy consumption worldwide. Ausubel and colleagues, sufficiently aware of this alternation, have built it into their forecasts. Figure 6 , which has been taken from their publication [13], shows three cycles of logistic growth followed by chaos: two depicted by data and one forecasted by the authors.

\section{Conclusions}

Chaos studies, as they have been carried out so far, focus on what happens after the niche is filled. The initial S-shaped rise is largely ignored by chaos scientists, who are only interested in phenomena with erratic fluctuations. Many real cases, however, like the production of coal and the world energy consumption shown earlier, display multiple periods of growth. New markets open new niches and the historical picture

\footnotetext{
${ }^{3}$ An early version of Figure 5 can be found in Marchetti [12]
} 


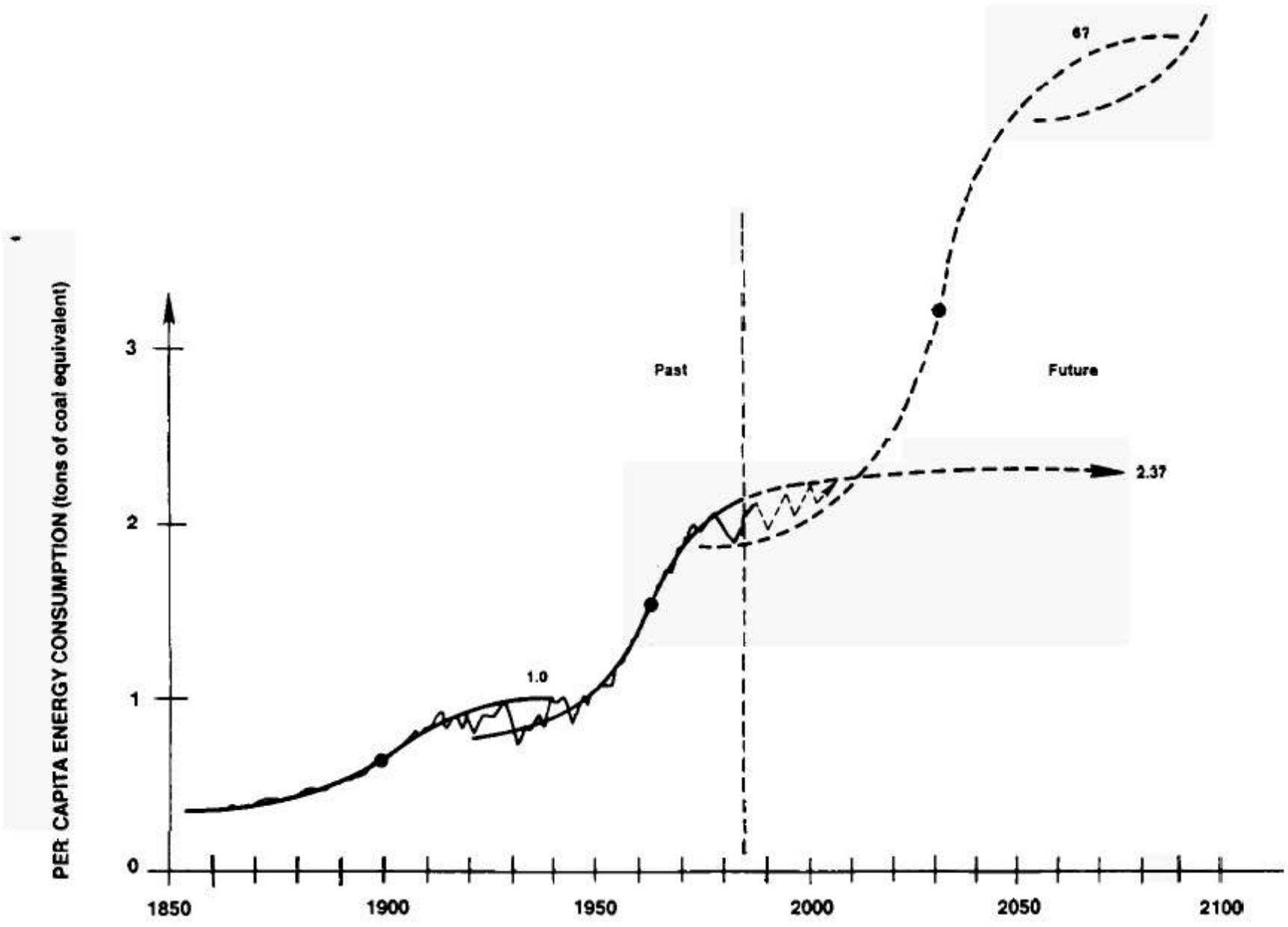

Fig. 6. The per capita annual energy consumption worldwide. The graph published by Ausubel at el. [13] shows data, logistic fits, and scenarios for the future.

becomes an alternation of logistic growth with periods of instability. The chaotic fluctuations belong to the end of a growth phase as much as to the beginning of the next one. Associating a peak with the past as an overshoot, or with the future as a precursor, is in some cases a simple question of taste.

The method described here for casting an $\mathrm{S}$ curve into a discrete form accounts for instabilities at both ends of logistic growth. One could reasonably expect that an upcoming growth phase in a new market niche will be heralded by precursors and, once installed, will proceed at an accelerated rhythm in the beginning. Moreover, exploring the mathematical formulation (through variation of the parameters) can teach us how the frequency and the importance of the precursors relate to the steepness and the size of the catching up, which in turn affects the instabilities expected at the top.

There is one final remark. A well-established $S$ curve will point to the time when chaotic oscillations should be expected; it is when the ceiling is being approached. In contrast, an entrenched chaos will reveal nothing about when the next growth phase will start. One has to locate the next growth phase from other considerations particular to each case. As suggested in the work of Ausubel et al. [13], the Kondratieff cycle is one way to position growth periods. In both examples shown earlier, namely, coal production in the United States and world energy consumption, there is coincidence between periods of steep rise in growth and a Kondratieff upswing, namely, 1884-1912 and 1940-1968. 


\section{References}

1. Peitgen, H. O., and Richter, P. H., The Beauty of Fractals, Springer-Verlag, Berlin, 1986.

2. Verhulst, P. F., Rechercher Mathématiques sur la Loi d'Accroissement de la Population, in Nouveaux Mémoires de l'Académie Royale des Sciences, et Belles-Lettres de Bruxelles, tome XVIII, 1-40 (1845).

3. Mandelbrot, B., The Fractal Geometry of Nature, Freeman, New York, 1977.

4. Modis, T., and Debecker, A., Determining the Services Life Cycle of Computers, in The Diffusion of Technologies and Social Behavior. N. Nakicenovic and A. Grubler, Springer-Verlag, Berlin, 1991.

5. Meade, N., Forecasting Using Growth Curves: An Adaptive Approach, Journal of the Operational Research Society, 36(12), 1985, pp 1103-1115.

6. Gleick, J., Chaos, Viking, New York, 1987, pp. 63-72.

7. Montroll, E. W., and Badger, W. W., Introduction to Quantitative Aspects of Social Phenomena, Gordon and Breach, New York, 1974.

8. Marchetti, C., The Automobile in a System Context: The Past 80 Years and the Next 20 Years, Technological Forecasting and Social Change, 23, 3-23 (1983).

9. Montrey, H., and Utterback, J., Current Status and Future of Structural Panels in the Wood Products Industry, Technological Forecasting and Social Change 38, 15-35, 1990.

10. Bureau of the Census, Statistical Abstract of the USA, Washington, DC, U.S. Government Printing Office, 1985-1990.

11. Bureau of the Census, Historical Statistics of the United States, Colonial Times to 1970, Washington, DC, U.S. Government Printing Office, 1975.

12. Marchetti, C., Energy Systems: The Broader Context, Technological Forecasting and Social Change 14 , 191-203, 1979.

13. Ausubel, J., Grübler, A., and Nakićenović, N., Carbon Dioxide Emissions in a Methane Economy, Climatic Change 12, 245-263, 1988. 